\title{
Empowerment e orientação empreendedora: Fundamentação teórica
}

\section{Empowerment and entrepreneurial orientation: Theoretical framework}

\author{
Orlando Lima Rua \\ Centro de Estudos Organizacionais e Sociais do P. Porto (CEOS-PP) e Unidade de \\ Investigação Aplicada em Gestão (UNIAG) \\ orua@iscap.ipp.pt \\ Sónia Rodrigues \\ Associação dos Institutos Superiores da Região do Norte de Portugal (APNOR) \\ s.andreiarodrigues@gmail.com
}

\begin{abstract}
Resumo
O objetivo fundamental deste estudo é definir o marco teórico da relação entre empowerment e orientação empreendedora, com vista a propor um modelo conceptual de investigação que possa vir a ser aplicado aos estabelecimentos hoteleiros da Região do Norte de Portugal. Para tal, utilizamos dados bibliográficos tendentes à delimitação teórica dos construtos estudados e a respetiva relação entre ambos. Espera-se que o presente estudo permita consolidar o empowerment como fator explicativo da orientação empreendedora, atendendo particularmente à realidade portuguesa.
\end{abstract}

Palavras-chave: empowerment; orientação empreendedora; setor hoteleiro; Norte de Portugal.

\begin{abstract}
The main purpose of this study is to develop a theoretical framework for the relationship between empowerment and entrepreneurial orientation, in order to propose a conceptual model that can be applied to the hospitality sector in the Northern Region of Portugal. To this end, we used bibliographical sources for the theoretical presentation of the two concepts and to explore their relationship. It is hoped that this study contributes to the consolidation of empowerment as an explanatory variable of entrepreneurial orientation, particularly in the Portuguese context.
\end{abstract}

Keywords: empowerment; entrepreneurial orientation; hospitality industry; Northern Portugal.

\section{Introdução}

As empresas inserem-se num ambiente em constante evolução onde o contexto de trabalho está exposto a intensas transformações oriundas das exigências do ambiente externo. A competitividade, produtividade e sustentabilidade surgem como principais desafios organizacionais, nos quais é necessário responder rápida e eficazmente, recorrendo a novos métodos de gestão sustentados em líderes ativos que operem mudanças profundas, estruturais e contínuas. É a partir desta premissa que o estudo do empowerment e da orientação empreendedora apresenta como denominador comum o capital humano. De acordo com Cardon e Stevens (2004), são os recursos humanos que ditam o sucesso ou fracasso de todas as organizações, por sua vez, Baron e Hannan (2002), afirmam que o conhecimento dos colaboradores representa uma mais-valia para a empresa na medida em

Dos Algarves: A Multidisciplinary e-Journal, 31- 2017.

ISBN 2182-5580 @ ESGHT - University of the Algarve, Portugal.

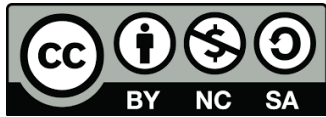

To cite this article: O.L. Rua e S. Rodrigues (2017). Empowerment e orientação empreendedora: Fundamentação teórica. Dos Algarves: A Multidisciplinary e-Journal, 31, 70-80. doi: 10.18089/DAMeJ.2017.31.5 
que cada um deles possui uma experiência própria e um saber empírico que resulta num melhor desempenho organizacional. Assim, os colaboradores deixam de ser encarados como um mero recurso produtivo e passam a assumir um papel fundamental no sucesso organizacional, sendo esse papel realçado no caso das organizações cuja atividade principal se prende com as prestações de serviços.

Samuel (2014) sustenta os benefícios económicos e sociais advindos da forte ligação do empowerment ao empreendedorismo, designadamente por via da criação e desenvolvimento de atividades geradoras de rendimento e emprego sustentável. Daqui deriva o desafio de qualificar indivíduos com orientação empreendedora, alinhados com uma filosofia de gestão e uma prática de tomada de decisão caraterizada pela inovação, proatividade e uma vontade de assumir riscos (Miller, 1983; Covin \& Slevin, 1989, 1991). O foco não é sobre o indivíduo, mas no processo de empreender (Wiklund, 1999). Porquanto, o empowerment é determinante para o processo empreendedor e para a formação de capital humano e não apenas na aquisição de ativos com o propósito de empreender (Karaan, 2006).

Neste contexto é incontornável capacitar os empreendedores para a inovação e criatividade, para começar algo sem que tenham um exemplo ou diretrizes políticas prévios, para a proatividade com vista à identificação de iniciativas que conduzem à ação sem que sejam obrigados a seguir determinada direção, para a assunção de riscos, vendo o indivíduo como parceiro com responsabilidades na tomada de decisão em novos projetos, potenciando assim o seu grau de controlo e inovação, tal significa uma mudança do paradigma (Ras \& Vermeulen, 2012).

Nas últimas décadas, o setor do turismo tem apresentado um crescimento contínuo; o seu o volume de negócios iguala o volume de exportações de petróleo, produtos alimentares ou até mesmo de automóveis. Este crescimento exponencial faz com que este setor se torne, cada vez mais, um fator-chave no desenvolvimento socioeconómico de cada país (WTO, 2015). Desta forma, pensamos ser relevante estudar o setor do turismo e especificamente a atividade económica da hotelaria, na qual a interação entre empresa-cliente é essencial para o sucesso empresarial, desempenhando os recursos humanos um papel crucial. De acordo com Arnett, Lavarie e McLane (2002), a forma como os colaboradores se sentem face ao seu trabalho pode fazer a diferença entre fazerem simplesmente o seu trabalho ou oferecerem um serviço excecional aos hóspedes, pois nesta indústria a satisfação do cliente encoraja a repetição e potencia a publicidade boca-aboca (Spinelli \& Canavos, 2000).

Apesar do setor dos serviços trazer vantagens no que diz respeito à empregabilidade, a rotatividade do pessoal, a sazonalidade, os salários baixos e contratação de pessoas com baixos níveis de qualificação continuam a ser alguns dos problemas que afetam a indústria hoteleira. Para contornar alguns destes problemas, Lashley e Lee-Ross (2003) afirmam que é necessário um entendimento claro das atitudes e comportamentos dos empregados de modo a que sejam implementadas estratégias que aumentem o bem-estar dos empregados, a produtividade, o comprometimento e o sucesso organizacional.

O empowerment não resolve todos os problemas organizacionais. Wilkinson (1998) sugere que é necessário um suporte organizacional sustentável para que a sua implementação seja benéfica quer para a organização, quer para os colaboradores. Yukl e Becker (2006) acrescentam que a aplicação do empowerment torna-se limitada quando a organização trabalha com operações rotineiras e serviços repetitivos. Robbins, Crino e Frendendall (2002) afirmam que o passo mais crítico no processo de empowerment é a criação de um ambiente 
de trabalho que promova a oportunidade de exercer uma gama completa de autoridade e poder, bem como a motivação dos colaboradores para adotar esse tipo de comportamentos.

A delegação de poderes está em voga no mercado de trabalho atual, pelo que o fornecimento de uma resposta rápida às alterações do mercado ditará o sucesso da organização, para tal é necessário o empowerment dos colaboradores e não a sua dependência. Cada organização é única e diferenciada de todas as demais, desta forma a implementação do empowerment terá de ajustar-se consoante as necessidades, contexto e cultura organizacionais de cada uma delas, correspondendo assim o empowerment a um construto multidimensional no qual são consideradas as atitudes dos líderes, as reações dos liderados e a forma como os trabalhos são estruturados (Honold, 1997).

O empreendedorismo é definido por Hisrich e Peters (1992) como o processo de criação de algo diferente, de valor, dedicando o tempo e o esforço necessários, assumindo os riscos financeiros, psicológicos e sociais, e recebendo as recompensas resultantes de satisfação monetária e pessoal. Os empreendedores são vistos por Schumpeter como indivíduos inovadores, capazes de introduzir novos produtos ou serviços, criar novos métodos de produção, encontrar novas fontes de matéria-prima e operar dentro de novos mercados (Robinson, Stimpson, Huefner, \& Hunt, 1991). Desde então, o empreendedorismo passou a estar relacionado com novos negócios, nos quais se insere a figura do empreendedor. Por outro lado, a orientação empreendedora associou-se ao processo de empreender, ou seja, aos métodos, práticas e estilos de tomada de decisão utilizados para agir de forma empreendedora (Lumpkin \& Dess, 1996). O fenómeno da orientação empreendedora como força motriz dos processos estratégicos das atividades empresariais tem merecido especial atenção na literatura sobre o empreendedorismo, sendo objeto de estudo de diversas pesquisas (Covin \& Walles, 2012).

Apesar da existência de estudos que relacionam estas variáveis e nos quais onde o foco principal são os recursos humanos (e.g. Brockbank, 1999; Hayton, 2003), a sua aplicação à indústria hoteleira do Norte de Portugal parece ser pioneira.

\section{Revisão de literatura}

\subsection{Empowerment}

Diversas organizações têm demonstrado especial interesse em abandonar as práticas de gestão conservadoras nas quais a centralização do poder e a falta de autonomia dos colaboradores impede o desenvolvimento e a valorização das organizações. Nesse sentido, começam a acreditar que a adoção de métodos que integrem a participação dos colaboradores com ideias e sugestões pode ser o caminho para o sucesso organizacional. Para tal é necessário um aumento da autonomia dos colaboradores no desempenho das suas funções, bem como um maior envolvimento na tomada de decisões na organização. O empowerment surge-nos assim como a ferramenta de gestão indicada para desenvolver este processo (Wall, Wood, \& Leach, 2004).

Começou então a emergir um elevado interesse na participação dos colaboradores nas tomadas de decisão das organizações e, na década de 80, já o envolvimento dos trabalhadores nas organizações em que estavam inseridos era notório, tendo surgido uma nova ferramenta de gestão intitulada de empowerment (Huq, 2010). Pfeiffer e Dunlop (1990) afirmam que esta ferramenta consiste na capacitação e na valorização do funcionário para que este contribua para a inovação e resolução de problemas no local de trabalho. O empowerment diz assim respeito à liberdade de escolha e ao poder dado aos funcionários pelos gestores de topo, de 
modo a promover a autonomia e responsabilidade no local de trabalho (Brymer, 1991). Cunningham e Hyman (1999) acrescentam que este é o caminho para conseguir o comprometimento dos colaboradores de modo a que contribuam para as decisões estratégicas com o objetivo de melhorar o desempenho da organização. Empowerment consiste assim no processo de dar aos colaboradores poder, liberdade e informação para que possam ajudar na tomada de decisões e participar ativamente na organização.

A abordagem de empowerment até aqui exposta surge associada a várias práticas de gestão que assentam na partilha do poder e na delegação de responsabilidades. No entanto, a sua conceptualização apresenta outra corrente de pesquisa assente numa vertente psicológica (Zhang \& Bartol, 2010). Conger e Kanugo (1988) sustentam que as-práticas de gestão que apelem ao empowerment, per si, não são suficientes para influenciar o comportamento dos indivíduos, é também necessário que estes se sintam capacitados. Nessa linha de investigação, o empowerment é entendido como um estado psicológico motivacional proveniente de uma necessidade intrínseca de autodeterminação e de uma crença na autoeficácia pessoal.

Thomas e Velthouse (1990) alargaram a definição inicial de Conger e Kanugo (1988), associando-lhe quatro dimensões cognitivas: significado, competência, autodeterminação e impacto que, quando relacionadas entre si, reforçam mutuamente a conceção de empowerment (Ro \& Chen, 2011). A avaliação positiva ou negativa destas quatro dimensões ditará o nível de motivação intrínseca, positivismo e autoconfiança com que o subordinado realizará a sua tarefa (Fernandez \& Moldogaziev, 2013).

Pelo exposto verificamos que o empowerment pode ser usado para descrever tanto um aspeto individual das pessoas como um aspeto organizacional das empresas. Por um lado, o empowerment psicológico traduz-se na reação dos colaboradores face às práticas de trabalho, por outro, o empowerment organizacional representa essas mesmas práticas de trabalho (Wilkinson, 1998). Torna-se, portanto, inevitável a abordagem das duas correntes de pesquisa como um só constructo, uma vez que o empowerment organizacional é consequência do empowerment psicológico.

Do ponto de vista organizacional, o empowerment assenta na partilha do poder, informações, recursos e recompensas por todos os níveis hierárquicos (Fernandez \& Moldogaziev, 2013), parecendo esta descentralização estar intimamente relacionada com a liderança visto que é nos líderes e gestores de topo que se inicia este processo.

No fundo, o conceito de empowerment tem subjacente a máxima de que os colaboradores são um recurso com conhecimento e experiência, capazes de diagnosticar, analisar e propor soluções para os problemas quotidianos (Wilkinson, 1998). Ainda segundo este autor, este envolvimento proporciona ganhos quer para as organizações, através do aumento do seu desempenho, quer para os colaboradores, potenciando a sua satisfação com o trabalho. Estes efeitos são evidenciados num conjunto crescente de pesquisas (Fernandez \& Moldogaziev, 2013).

Apesar da abundante investigação em torno da conceptualização de empowerment, não vislumbrámos nenhum suporte que identificasse com clareza e precisão os seus construtos (Yukl \& Becker, 2006). Enquanto vertente psicológica, o empowerment é tipicamente associado a uma compilação de fatores desenvolvidos por Thomas e Velthouse (1990), a saber:

1. O significado refere-se à concretização de um objetivo segundo uma avaliação intrínseca feita pelo indivíduo. Esta dimensão é descrita como o motor do empowerment 
no sentido de dar energia ao capital humano de uma organização para trabalhar com objetivos comuns (Spreitzer, Kizilos, \& Nason, 1997);

2. A competência diz respeito à crença que o indivíduo tem em relação à sua capacidade para realizar as suas tarefas com habilidade e sucesso;

3. A autodeterminação refere-se à autonomia que um indivíduo tem para decidir a forma como vai realizar as suas tarefas (Zhang \& Bartol, 2010). É uma característica de pessoas determinadas, capazes de grandes feitos de mudança. Para alguns autores a autodeterminação é um ponto crucial do empowerment (Yukl \& Becker, 2006);

4. Por fim, o impacto expõe a intensidade com que um indivíduo pode influenciar os resultados estratégicos, administrativos ou operacionais no seu trabalho (Ro \& Chen, 2011).

Não está claro se as dimensões supra referenciadas são as mesmas para o empowerment organizacional, pelo que é necessário uma maior precisão na sua definição e medição (Yukl \& Becker, 2006). No entanto, sendo o empowerment organizacional uma consequência do psicológico, torna-se lógico recorrer a estas dimensões para a sua medição (Wilkinson, 1998).

\subsection{Orientação empreendedora}

As empresas estão inseridas num ambiente empresarial altamente competitivo e dinâmico no qual é imperativo adotar estratégias empreendedoras capazes de levar as organizações a bom porto (Davis, Bell, Payne, \& Kreiser, 2010). Neste seguimento, a orientação empreendedora emerge como um conceito merecedor de especial atenção, capaz de investigar o espírito empreendedor das organizações, bem como a sua influência sobre decisões estratégicas e desempenho organizacional (Rauch, Wiklund, Lumpkin, \& Frese, 2009).

Inicialmente, o conceito de orientação empreendedora surgia associado a uma qualidade atribuída ao gerente ou proprietário, quando este era capaz de desafiar a incerteza do ambiente através da implementação de ações corporativas. Miller (1983) desenvolveu a interpretação deste conceito, tornando-o mais completo, sugerindo que este fosse interpretado como uma caraterística das empresas, para além do desempenho de um único indivíduo, principalmente tratando-se de uma grande empresa com processos complexos. De acordo com este autor, uma empresa desenvolve orientação empreendedora quando apresenta constantemente inovações de produtos e de mercados, assume riscos e se comporta de forma proativa (Davis et al., 2010). Esta definição surgiu a partir da definição de empreendedorismo, que desde a década de 90 começou a ser tratada como uma área de pesquisa distinta do empreendedorismo.

Naturalmente, a orientação empreendedora nas organizações exige mais do que a simples prevalência de valores empreendedores, oriundos da tendência individual dos gestores de topo de uma empresa (Kreiser, Marino, \& Weaver, 2002). É necessário que os comportamentos empreendedores sejam transmitidos a todos os níveis da organização, desde a gestão de topo até à gestão operacional (Covin \& Slevin, 1998). Assim, o comportamento empreendedor a nível individual pode afetar toda a ação organizacional e, em muitos casos, os comportamentos empreendedores, individual e organizacional, podem ser muito semelhantes (Wiklund, 1999).

A orientação empreendedora emerge assim como uma perspetiva de escolha estratégica, na qual as oportunidades de negócio podem ser empreendidas com sucesso de forma intencional. Este caráter comportamental faz com que a intensidade da orientação empreendedora varie de organização para organização (Lumpkin \& Dess, 1996). 
Embora tenha havido diferentes propostas para reconhecer as dimensões inerentes à orientação empreendedora, o trabalho de Miller (1983) é um dos primeiros a tratar esta temática, tendo identificado três dimensões que permitem caraterizar e verificar a presença da orientação empreendedora nas organizações, sendo elas: a proatividade, a inovação e a assunção de riscos.

Apesar de na literatura serem consideradas mais dimensões, nomeadamente a agressividade e a autonomia, grande parte dos autores tem adotado a abordagem proposta por Miller nos seus trabalhos (Lumpkin \& Dess, 1996). Esta abordagem trata a orientação empreendedora como um construto unidimensional, no qual as três dimensões (proatividade, inovação e assunção de riscos) devem estar presentes em certo grau numa organização para que esta seja considerada empreendedora, por outro lado, uma organização não empreendedora é aquela que inova pouco, é avessa ao risco e mantém uma atitude passiva perante os seus concorrentes (Miller, 1983).

A dimensão da inovação reflete a tendência de uma organização para participar e apoiar novas ideias, novidades, processos experimentais e criativos que possam resultar em novos produtos, serviços ou processos (Lumpkin \& Dess, 1996). Os recursos financeiros investidos na inovação e o nível de comprometimento dos recursos humanos com as atividades inovadoras ditará o grau de inovação de uma organização (Covin \& Slevin, 1989). Sendo certo que todos os tipos de inovações envolvem uma série de esforços, sejam eles tecnológicos, administrativos, de produtos ou mercados, independentemente do tipo de inovação adotado, são as empresas mais inovadores que tendem a superar situações mais instáveis. Desta forma, existe o consenso de que esta dimensão é a mais importante no âmbito da orientação empreendedora, uma vez que determina um meio pelo qual as organizações podem alcançar vantagem competitiva e promover o crescimento da organização (Dess \& Lumpkin, 2005).

A proatividade inclui a busca ativa de novas oportunidades de negócio e a antecipação de mercados emergentes (Lumpkin \& Dess, 1996). Miller (1983) e Covin e Slevin (1989) referem que a proatividade pode ser avaliada por meio da tendência que a organização tem para estar à frente no desenvolvimento de novos produtos e tecnologias e na introdução de novos produtos ou serviços, ao contrário de simplesmente acompanhar a evolução do mercado. A proatividade é considerada mais importante para as empresas em estágios iniciais de desenvolvimento do que nas indústrias mais maduras, desta forma, esta dimensão pode ser vista como um contínuo (Lumpkin \& Dess, 1996), capaz de moldar o ambiente empresarial no sentido de alcançar vantagem competitiva (Miller, 1983).

A assunção de riscos refere-se à disposição dos gestores para comprometer recursos significativos com oportunidades incertas (Miller, 1983). Na literatura menos recente, a assunção de riscos era apresentada como uma caraterística individual do empreendedor, que, quando aplicada à organização, reflete o comportamento de assumir riscos, tais como o de assumir grandes compromissos financeiros visando obter altos retornos (Lumpkin \& Dess, 1996). A assunção de riscos centra-se na tendência da organização para se comprometer-se com projetos de risco e na preferência dos empreendedores para atuar de forma ousada ao mesmo tempo que cautelosa para alcançar os objetivos organizacionais (Miller, 1983).

\subsection{Modelo e hipóteses de investigação}


A orientação empreendedora é uma estratégia que requer um grande investimento por parte das empresas. É necessário construir, incutir, promover e manter os comportamentos empreendedores para alcançar o sucesso empresarial (Arham \& Muenjohn, 2012).

O empowerment é considerado o motor de uma cultura de inovação, de criatividade e iniciativa (Brockbank, 1999), potenciador de um espírito empreendedor na organização. Num estudo realizado a 99 PME americanas com a finalidade de verificar de que forma uma organização poderia promover o desempenho empreendedor, Hayton (2003), concluiu que o empowerment é uma das ferramentas de gestão que pode ser utilizada na melhoria da orientação empreendedora. Segundo este autor, a descentralização do poder estimula o compromisso do trabalhador, a cooperação, a partilha de conhecimento e as atividades voluntárias, sustentando que a melhoria do espírito empreendedor de uma organização passa pela descentralização do poder para que todos os colaboradores se envolvam entusiasticamente nos desafios do seu trabalho.

As questões utilizadas para avaliação do empowerment foram extraídas do estudo de Spreitzer (1995), que têm por base as dimensões propostas por Thomas e Velthouse (1990). Apesar desta pertinência, ainda não existe nenhuma adaptação do instrumento para português, pelo que tentamos assegurar a equivalência conceptual dos itens recorrendo a uma tradução direta dos itens originais, por conseguinte, o instrumento final é constituído por doze itens, que medem as quatro dimensões propostas:

1. Significado: (SIG1) O trabalho que eu faço é muito importante para mim; (SIG2) As minhas tarefas têm um significado pessoal para mim; (SIG3) O trabalho que eu faço tem significado para mim;

2. Competência: (COMP1)Tenho confiança nas minhas competências para executar o meu trabalho; (COMP2) Tenho confiança nas minhas competências para realizar as minhas atividades; (COMP3) Domino as competências necessárias para executar o meu trabalho;

3. Autodeterminação: (ADET1) Tenho autonomia significativa para determinar como fazer o meu trabalho; (ADET2) Posso decidir por mim como proceder para fazer o meu trabalho; (ADET3) Tenho oportunidade de realizar o meu trabalho de forma livre e independente;

4. Impacto: (IMP1) Aquilo que eu faço tem um grande impacto no meu departamento; (IMP2) Tenho um grande controlo sobre o que acontece no meu departamento; (IMP3) Tenho influência significativa sobre o que acontece no meu departamento.

Por fim, com o intuito de avaliar a orientação empreendedora recorreu-se à adaptação do estudo de Rodrigues (2004) baseado originalmente na escala proposta por Covin e Slevin (1989) constituída por nove itens: três para a inovação, três para proatividade e três para a assunção do risco. Esta divisão vai ao encontro das dimensões da orientação empreendedora propostas por Miller (1983), anteriormente referidas:

a) Inovação: (INOV1) Em que medida, nos últimos cinco anos, a empresa tem valorizado a inovação, investigação e desenvolvimento (I\&D) e a liderança tecnológica em detrimento da comercialização de produtos ou serviços já existentes na empresa, cujo valor é conhecido; (INOV2) Em que medida, nos últimos cinco anos, a empresa lançou novas linhas de produtos; e (INOV3) Em que medida, nos últimos cinco anos, a empresa efetuou mudanças significativas nas linhas de produtos; 
b) Proatividade: (PROAT1) Relativamente à concorrência, em que medida a empresa inicia ações a que os concorrentes respondem em vez de responder às ações iniciadas por eles; (PROAT2) Relativamente à concorrência, em que medida a empresa é a primeira a introduzir novos produtos, serviços, técnicas de gestão, tecnologias operativas, etc.; e (PROAT3) Relativamente à concorrência, em que medida a empresa procura ativamente que os seus concorrentes saiam do mercado;

c) Assunção de risco: (ASRIS1) Em que medida na empresa os gestores do topo preferem projetos de alto risco (com elevado grau de incerteza da rentabilidade); (ASRIS2) Em que medida na empresa se acredita que, dada a natureza do mercado, são necessárias ações arrojadas e de grande impacto para alcançar os objetivos da empresa; e (ASRIS3) Em que medida na empresa se adota uma postura arrojada e agressiva, no sentido de explorar ao máximo a probabilidade de fortes oportunidades.

A totalidade dos itens apresentados foi avaliada tendo por base uma escala de Likert de sete pontos, onde o um significa "discordo totalmente" e o sete "concordo totalmente".

Do referido deriva a formulação da seguinte hipótese de investigação:

H1: O empowerment tem um efeito positivo e significativo na orientação empreendedora.

Na Figura 1 apresenta-se o modelo teórico de investigação, do qual constam as dimensões do empowerment como variáveis latentes da orientação empreendedora.

Figura 1. Modelo teórico de investigação

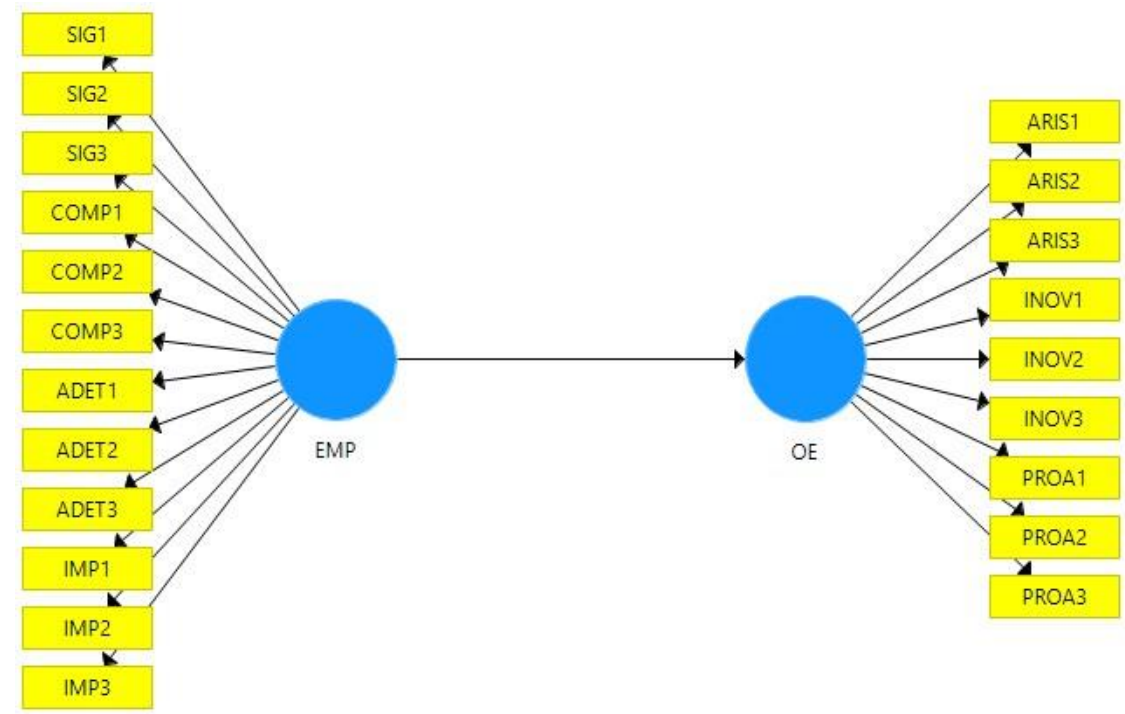

Legenda: EMP - Empowerment (SIG - Significado; COMP - Competência; ADET Autodeterminação; IMP - Impacto); OE - Orientação empreendedora (INOV - Inovação; PROAT - Proatividade; ARISC - Assunção de riscos).

\section{Considerações finais}

Neste estudo pretende-se desenvolver um marco teórico que explique a relação entre empowerment e orientação empreendedora.

Tendo por base uma pesquisa bibliográfica, verificou-se a existência de uma lacuna no estudo do conceito de empowerment enquanto fator explicativo da orientação 
empreendedora. Desde modo, esta investigação visou, por um lado, a delimitação teórica dos construtos e, por outro, propor um modelo concetual de investigação, com vista à sua aplicação aos estabelecimentos hoteleiros da Região do Norte de Portugal.

Ainda que este setor apresente crescimentos notórios quer do ponto de vista da empregabilidade quer do ponto de vista económico, os gestores ainda têm um longo caminho a percorrer para fomentar o espírito empreendedor e o empowerment dos seus seguidores. É necessário apostar na capacitação dos funcionários e em estruturas hierárquicas capazes de responder eficazmente às necessidades pessoais e organizacionais, de acordo com Agência Lusa (2015), só assim haverá qualidade no serviço prestado e na capacidade de resposta ao aumento de turistas, que se perspetiva nos próximos anos.

O estudo empírico a realizar compreenderá o recurso a técnicas estatísticas com vista a realizar as respetivas análises descritiva e inferencial. Destacamos a aplicação do modelo de equações estruturais, que consiste numa regressão múltipla com indicadores refletivos que se apresentam como um reflexo do constructo teórico não observado, dando lugar às variáveis observadas ou medidas, cujo objetivo é reforçar a relação de influência entre os construtos (Marôco, 2010).

O Partial Least Squares (PLS) é a técnica que melhor se adapta a aplicações preditivas (análise exploratória) e desenvolvimento da teoria quando esta não está solidamente desenvolvida (Cepeda \& Roldán, 2004), bem como maximiza a variância explicada das variáveis dependentes (latentes ou observadas, ou ambas) e estima modelos estruturais com amostras pequenas (Chin \& Newsted, 1999; Reinartz, Haenlein \& Henseler, 2009). Por outro lado, estima modelos com medidas refletivas e formativas sem problema de identificação (Chin, 2010).

Porquanto, espera-se com esta pesquisa contribuir para o desenvolvimento da literatura nas áreas do empowerment e da orientação empreendedora. Em Portugal não foram identificados trabalhos que interliguem estas duas áreas de estudo, pelo que esta pesquisa permitirá observar a realidade portuguesa neste âmbito.

\section{Referências}

Arham, A. \& Muenjohn, N. (2012). Leadership and organisational performance in Malaysian SMEs: The mediating role of entrepreneurial orientation. Proceedings of Business and Information, 31-41.

Arnett, D., Lavarie, D. \& McLane, C. (2002). Using job satisfaction and pride as internal-marketing tools. Cornell Hotel and Restaurant Administration Quarterly, 43(2), 87-96.

Baron, J. \& Hannan, T. (2002). Organizational blueprints for success in high-tech start-ups: Lessons from the Stanford project on emerging companies. California Management Review, 44 (3), 8-36.

Brockbank, W. (1999). If HR were really strategically proactive: Present and future in RH's contribution to competitive advantage. Human Resource Management, 38(4), 337-352. doi: 10.1002/(SICl)1099050X(199924)38:4<337::AID-HRM8>3.0.CO;2-5.

Brymer, R. A. (1991). Employee empowerment: A guest-driven leadership strategy. Cornell Hotel and Restaurant Administration Quarterly, 32(1), 58-68. doi: 10.1177/001088049103200116.

Cardon, M. \& Stevens, C. (2004). Managing human resources in small organization: What do we know? Human Resources Management Review, 14(3), 295-323.

Cepeda, G. \& Roldán, J. L. (2004). Aplicando en la práctica la técnica PLS en la administración de empresas. XIV Congreso Nacional ACEDE, 74-78.

Chin, W. W. (2010). How to write up and report PLS analyses. In V. Esposito Vinzi, W. W. Chin, J. Henseler \& H. Wang (Eds.), Handbook of partial least squares: Concepts, methods and applications (pp. 655690). Berlin, Germany: Springer-Verlag.

Chin, W. W. \& Newsted, P. R. (1999). Structural equation modeling analysis with small samples using partial least squares. In R. Hoyle (Eds.), Statistical strategies for small sample research (pp. 307-341). Thousand Oaks, CA: Sage Publications. 
Conger, J. A., \& Kanugo, R. N. (1988). The empowerment process: Integrating theory and practice. Academy of Management Review, 13 (3), 471-482.

Covin, J. G. \& Slevin, D. P. (1989). Strategic management of small firms in hostile and beging environments. Strategic Management Journal, 10(1), 75-87. doi: 10.1002/smj.4250100107.

Covin, J.G. \& Slevin, D. P. (1991). A conceptual model of entrepreneurship as firm behavior. Entrepreneurship: Theory \& Practice, 16(1), 7-25.

Covin, J. G. \& Slevin, D. P. (1998). The influence of organization structure on the utility of an entrepreneurial top management style. Journal oh Management Studies, 25(3), 217-234. doi: 10.1111/j.1467-6486.1988.tbooo33.x.

Covin, J. G. \& Walles, J. (2012). The measurement of entrepreneurial orientation. Entrepreneurship Theory and Practice, 36(4), 677-702. doi: 10.1111/j.1540-6520.2010.00432.x.

Cunningham, I. \& Hyman, J. (1999). The poverty of empowerment? A critical case study. Personnel Review, 28 (3), 192-207. doi.org/10.1108/00483489910264589.

Davis, J., Bell, R., Payne, G. \& Kreiser, P. (2010). Entrepreneurial orientation and firm performance: The moderating role of managerial power. American Journal of Business, 25(2), 41-54. doi: 10.1108/19355181201000009.

Dess, G. G. \& Lumpkin, G. T. (2005). The role of entrepreneurial orientation in stimulating effective corporate entrepreneurship. Academy of Management Perspect, 19(1), 147-156. doi: 10.5465/AME.2005.15841975

Fernandez, S. \& Moldogaziev, T. (2013). Employee empowerment, employee attitudes and performance: Testing s causal model. Public Administration Review, 73(3), 490-506. doi: $10.1111 /$ puar.12049

Hayton, J. C. (2003). Strategic human capital management in SME's: An empirical study of entreprenurial performance. Human Resource Management Journal, 42(4), 375-391. doi: 10.1002/hrm.10096.

Hisrich, R. \& Peters, M. (1992). Entrepreneurship: Starting, developing, and managing a new entreprise. Boston: Irwin.

Honold, L. (1997). A review of the literature on empoloyee empowerment. Empowerment in Organizations, 5 (4), 202-212. doi: 10.1108/14634449710195471.

Huq, R. (2010). Employee Empowerment: The rhetoric \& the reality (5th ed.). Axminster, U.K.: Triarchy Press.

Karaan, M. (2006). Re-imagining a future for South African agriculture, Agrekon, 45(3), 245-260. doi: 10.1080/03031853.2006.9523746

Kreiser, P. Marino, L. \& Weaver, M. (2002). Assessing the psychometric properties of the entrepreneurial orientation scale: A multi-country analysis. Entrepreneurship Theory and Practice, 26(4) 71-95.

Lashley, C. \& Lee-Ross, D. (2003). Organization behavior for leisure services. Oxford: Elsevier ButterworthHeinemann.

Lumpkin, G. T. \& Dess, G. G. (1996). Clarifying the entrepreneurial orientation construct and linking it to performance. The Academic of Management Review, 21(1), 135-172.

Lusa (2015, 21 de junho). Emprego no turismo do Porto cresce em parte à custa da precariedade e clandestinidade. Disponivel em http://www.rtp.pt/noticias/economia/emprego-no-turismo-doporto-cresce-em-parte-acusta-da-precariedade-e-clandestinidade_n838430.

Marôco, J. (2011). Análise de equações estruturais: Fundamentos teóricos, software \& aplicações. Pêro Pinheiro: ReportNumber, Lda.

Miller, D. (1983). The correlates of entrepreneurship in three types of firms. Management Science, 29(7), 770-791. doi: 10.1287/mnsc.29.7.770.

Pfeiffer, I. \& Dunlop, J. (1990). Increasing productivity through empowerment. Supervisory Management, 8-17.

Ras, P. J. \& Vermeulen, W. J. V. (2012). Innovative business cases in the South Africa table grape and wine industryies: Developing the concept of empowerment entrepreneurship. World Review of Entrepreneurship, Management and Sustainable Development, 8(4), 456-477. doi: 10.1504/WREMSD.2012.050301.

Rauch, A., Wiklund, J., Lumpkin, G. \& Frese, M. (2009). Entrepreneurial orientation and business performance: An assessment of past research and suggestions for the future. Entrepreneurial Theory and Practice, 33 (3), 761-787. doi: 10.1111/j.1540-6520.2009.00308.x

Reinartz, W., Haenlein, M. \& Henseler, J. (2009). An empirical comparison of the efficacy of covariancebased and variance-based SEM. International Journal of Research in Marketing, 26(4), 332-344. doi: 10.1016/j.jiresmar.2009.08.001. 
Ro, H. \& Chen, P. (2011). Empowerment in hospitality organizations: Customer orientation and organizational support. International Journal of Hospitality Management, 30(2), 422-428. doi: 10.1016/j.ijhm.2010.09.003.

Robbins, T., Crino, M. \& Frendendall, L. (2002). An integrative model of the empowerment. Human Resource Management Review, 12, 419-443.

Robinson, P., Stimpson, J., Huefner, J. \& Hunt, H. (1991). An attitude approach to the prediction of entrepreneurship. Entrepreneurship Theory \& Practice, 15(4), 13-31.

Rodrigues, R. (2004). Orientação para o mercado, orientação empreendedora e desempenho nas PME industriais: Exploração da relação entre marketing e empreendedorismo. Tese de Doutoramento (não publicada). Universidade da Beira Interior, Covilhã.

Samuel, L. (2014). O contributo do empreendedorismo feminino no empoderamento socioeconómico da mulher, estudo de caso (pemba, moçambique). Revista Elelectrónica de Investigação e Desenvolvimento, 2, 1-17.

Spinelli, M. \& Canavos, G. (2000). Investigating the relationship between employee satisfaction and guest satisfaction. Cornell Hotel and Restaurant Administration Quarterly, 41(6), 29-33.

Spreitzer, G. M. (1995). Psychological empowerment in the workplace: Dimensions, measurement and validation. Academy of Management Journal, 38(5), $1442-1465$.

Spreitzer, G. M., Kizilos, M. A. \& Nason, S. W. (1997). A dimensional analysis of the relationship between psychological empowerment and effectiveness satisfacion, and strain. Journal of Management, 23(5), 679-704. doi.org/10.1177/014920639702300504.

Thomas, K. \& Velthouse, B. (1990). Cognitive elements of empowerment: An interpretive model of intrinsic task motivation. Academy of Management Review, 15(4), 666-681.

Wall, T., Wood, S. \& Leach, D. (2004). Empowerment and performance. International Review of Industrial and Organizational Psychology, 19, 1-44.

Wiklund, J. (1999). The sustainability of the entrepreneurial orientation performance relationship. Entrepreneurship: Theory \& Practice, 24(1), 39-50.

Wilkinson, A. (1998). Empowerment: Theory and practice. Personnel Review, 27(1), 40-56. doi: $10.1108 / 00483489810368549$

WTO (2015). Why Tourism? (http://www2.unwto.org/content/whytourism).

Yukl, G. A., \& Becker, W. S. (2006). Effective empowerment in organizations. Organization Management Journal, 3(3), 210-231. doi: 10.1057/omj.2006.20.

Zhang, X. \& Bartol, K. (2010). Linking empowering leadership and employee creativity: The influence of psychological empowerment, intrinsic motivation, and creative process engagement. Academy of Management Journal, 53(1), 107-128. doi: 10.1108/dlo.2010.08124ead.007

ORLANDO LIMA RUA é professor Adjunto na área científica de Gestão no Instituto Superior de Contabilidade e Administração do Porto (ISCAP), do Politécnico do Porto. É doutor europeu em Economia e Gestão de Empresas (especialização em organização de empresas). É investigador efetivo do Centro de Estudos Organizacionais e Sociais do P. Porto (CEOS.PP) / ISCAP e investigador integrado na Unidade de Investigação Aplicada em Gestão (UNIAG) da Associação dos Institutos Superiores da Região do Norte de Portugal (APNOR). Os seus trabalhos têm sido publicados em livros, capítulos de livros, revistas e conferências internacionais nas áreas do empreendedorismo, inovação, estratégia e recursos humanos. Endereço institucional: Politécnico do Porto / ISCAP, Rua Jaime Lopes Amorim, s/n, 4465-004 S. Mamede de Infesta, Portugal.

SÓNIA RODRIGUES é licenciada em Engenharia Alimentar e mestre em Gestão das Organizações (Ramo de Gestão de Empresas), pela Associação dos Institutos Superiores da Região do Norte de Portugal e Politécnico do Porto / ISCAP. Iniciou a sua atividade profissional em 2013 como formadora de Sistemas HACCP e Higiene e Segurança Alimentar. Em 2015, iniciou o seu percurso na área da gestão hoteleira. Em agosto de 2017 integra uma equipa de controlo de qualidade num projeto relacionado com a reciclagem de óleos usados. Endereço institucional: Instituto Superior de Administração e Contabilidade do Porto, Politécnico do Porto, Rua Jaime Lopes Amorim, 4465-004 Matosinhos, Portugal.

Submetido em 15 janeiro 2017

Aceite em 5 novembro 2017 